\title{
Numerical and Experimental Investigation of Near-Field Mixing in Parallel Dual Round Jets
}

\author{
Xie Zheng, Xie Jian, Jiang Wei, and Du Wenzheng \\ Xian High Technology Research Institute, Xian 710025, China \\ Correspondence should be addressed to Xie Zheng; xiez19891121@163.com
}

Received 14 March 2016; Revised 9 June 2016; Accepted 12 July 2016

Academic Editor: William W. Liou

Copyright (c) 2016 Xie Zheng et al. This is an open access article distributed under the Creative Commons Attribution License, which permits unrestricted use, distribution, and reproduction in any medium, provided the original work is properly cited.

\begin{abstract}
Parallel underexpanded round jets system has been widely used in engineering applications, and the flow field structures are very complex because of the jets interaction. In this paper, we studied the near-field mixing phenomenon in parallel dual underexpanded jets numerically by solving the Reynolds-Averaged Navier-Stokes Equations. The numerical results agree well with experimental data acquired by particle image velocimetry. Similar to plane jets, to some degree, two round jets are deflected towards the dual nozzle symmetry plane; the flow field can also be divided into three regions. Meanwhile, attempts have been made to predict merge point and combine point locations on certain cross profile of computational domain by correlating them with jet spacing and jet pressure ratio. The jet spacing plays an important role in jets interaction, and jet interaction decreases with the increase in jet spacing. The jets interaction in terms of merge (combine) point and pressure varies significantly while the jet spacing differs. Additionally, as pressure ratio increases, the effect of jet interaction decreases, and the merge (combine) point location moves downstream.
\end{abstract}

\section{Introduction}

Parallel jets issuing from adjacent nozzles into still surroundings gradually merge into a single jet at a certain distance downstream, which has been widely used in many engineering applications such as vertical take-off and landing of airplanes, fuel-injection systems, and supply devices in ventilation systems $[1,2]$. In fact, there has been significant research effort dedicated to the coupling relationship of the jets [3-6]. However, coupling relationships of the jets are not fully understood yet. The previous reports reveal that three relevant regions of the flow field in axial direction were identified for the two-plane parallel jets [7-9]. Figure 1 shows the general flow field of two-plane parallel jets. The first region is converging region from nozzle exit to the point where the inner shear layers of the jets merge (termed as the merge point). At the merge point the velocity in the jet flow direction on the symmetry plane is zero and the pressure reaches the highest value $[10,11]$. The location where the velocity at the symmetry plane reaches its maximum is called the combined point and denotes that the merging region ends and the combined region begins $[12,13]$. The combined region is downstream of the combined point where the two jets begin to resemble a self-similar single jet $[6,14-16]$.

However, for the round nozzles, the three-dimensional flow structures need to be taken into consideration, and jets will interact and the position of maximum velocity will shift from the symmetry axis to the symmetry plane between the nozzles [17]. Numerical simulations of two round jets by Allen and Smith [18] indicated that the results of the standard $k-\varepsilon$ model are consistent with experimental data for parallel jets with low inlets. Harima et al. $[3,19]$ showed that the widely used turbulence models (RNG $k-\varepsilon$ model and realizable $k-\varepsilon$ model) can be used to predict mean velocity and turbulence properties in the near zone of interacting confluent round jets with low inlets precisely.

Wang [20] researched on the based flow for four-engine clustered nozzle, where a standard two-equation turbulence model was used to describe the turbulence, and a pressure inlet condition was imposed on nozzle inlet plane. The numerical results coincided well with the experimental results.

Although confluent jet phenomenon has been a topic of great interest to researchers over the past decade, there 


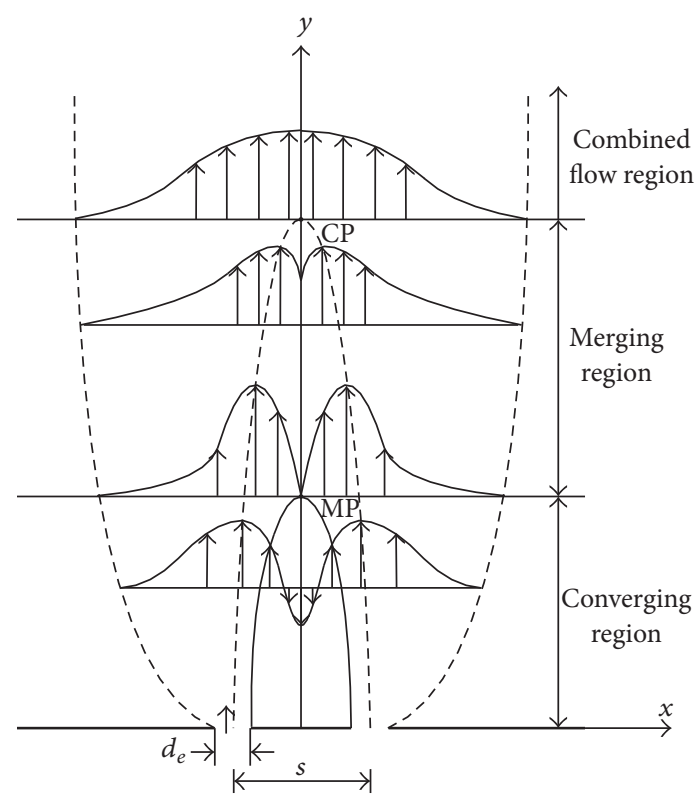

FIGURE 1: General flow field of two-plane parallel jets.

TABLE 1: Conditions for PIV measurement.

\begin{tabular}{lcc}
\hline Tests & $P_{0} / P_{b}$ & $s / d_{e}$ \\
\hline $1 \sim 8$ & 3.4 & $2,3,4,6,10,13,16,20$ \\
$9 \sim 16$ & 6.8 & \\
$17 \sim 24$ & 8.1 & \\
\hline
\end{tabular}

remain few literatures on prediction for the critical mixing regions (such as the merge point and the combine point) of parallel dual underexpanded jets. Furthermore, experimental investigations on the confluence of parallel dual underexpanded jets are sparse. This study is focused on near-field mixing phenomenon of dual underexpanded jets, and the PIV measurement and numerical simulation are used to study the jet interaction and its resulting characteristics. The main objectives are to gain insights into the complex phenomena occurring in this near-zone region and to obtain accurate and detailed experimental data that can be used to verify the numerical results. The PIV measurement and numerical simulation are used to study the jet interaction and its resulting characteristics. Furthermore, the effect of jet-to-jet spacing $\left(s / d_{e}\right)$ and the jet pressure ratio have been investigated. The jet pressure ratio has value of total pressure $P_{0}$ in the settling chamber divided by back pressure $P_{b} . P_{0} / P_{b}$ are $3.4,6.8$, and 8.1 in this study, respectively.

\section{Experimental Procedures}

Table 1 shows all the tests conducted in the experimental setup. High pressure gas at ambient temperature $(300 \mathrm{~K})$ is discharged into enclosure through a pair of converging circular nozzles. According to the jet pressure ratio, we divided the tests into three groups by using eight converging circular nozzles plates.
2.1. Experimental Setup. Figure 2 illustrates the schematic diagram of experiment setup. A controllable centrifugal fan accelerates air which first passes through a rotary flow meter and then flows towards the test chamber through square supply channels, bends, a diffuser, a settling chamber, and a contraction. The settling chamber is equipped with meshes and honeycombs in different sizes. A pressure regulating valve was used to control the settling chamber static pressure. The settling chamber is followed by a converging circular nozzles plate. From the converging circular nozzles plate, the jets issue into an enclosure with dimensions $0.6 \times 0.6 \times 1.2 \mathrm{~m}^{3}$ $(H \times W \times L)$. The enclosure serves to avoid disturbances from laboratory air motion and to ensure equal concentration of flow tracer particles in the jet fluid and in the surrounding fluid. The enclosure is equipped with two high transmittance optical windows so that no measurement equipment needs to be placed inside the enclosure.

2.2. Converging Circular Nozzles Plate Geometry. The sonic orifice is a conical convergent nozzle with a finite length, and the sonic nozzle profile is designed and shown in Figure 3. Its conical convergence angle is 12.52 degrees; the jet inlet diameter is $10 \mathrm{~mm}$; and the whole length is $16.5 \mathrm{~mm}$. The nozzle distance $s$ divided by $d_{e}, s / d_{e}$, ranges from 2.0 to 20.0.

2.3. PIV Measurement. As shown in Figure 2, the PIV measurement is performed in only one plane (nozzle center plane $z=0)$. The range in $y$-direction covers the area from the nozzle exit downstream $\left(y=1.2 d_{e}\right)$ up to a maximum distance of $y / d_{e}=80$. The PIV system comprises a New Wave Solo-II Nd:YAG twin cavity laser $(30 \mathrm{~mJ}$ per pulse at $15 \mathrm{~Hz}$ ) and a PCO Sensicam ${ }^{\mathrm{TM}}$ thermoelectrically cooled CCD camera $(1280 \times 1024$ px2, 12 bits $)$ with a $28 \mathrm{~mm}$ lens. A glycolwater aerosol is used for seeding, with a mean particle diameter of $0.25 \mu \mathrm{m}$. Customized optics is used to generate a $0.3 \mathrm{~mm}$ thick light sheet aligned as shown in Figure 3. The CCD camera is mounted perpendicularly to the light sheet. The raw images were postprocessed with the commercial software $\mathrm{DaVis}$ 7.2.2. The seeding density is high enough to assume an order of magnitude displacement uncertainty of 0.1 pixels [21], which yields an uncertainty in the velocity field between $1 \%$ for the high velocity region and $20 \%$ for the low velocity regions. According to the practical guidelines by Adrian and Westerweel [22], the uncertainty of the instantaneous velocity field is estimated within $\pm 3 \%$. The maximum statistical error, due to the finite number of samples (with $95 \%$ confidence), is less than $\pm 1.4 \%$ for mean velocities and less than $\pm 0.8 \%$ and $\pm 0.3 \%$ for normal and shear Reynolds stresses.

\section{Numerical Investigations}

3.1. Physical Model and Boundaries Condition. Figure 4 shows the computational domain of parallel double nozzles model measuring $40 d_{e} \times 60 d_{e} \times 80 d_{e}$, which includes the nozzles zones. The position of the origin is the center of the double nozzles outlet, and then Cartesian coordinates are presented. The no-slip and adiabatic boundary conditions are applied to the wall; the pressure outlet condition is applied to the computational domain outlet; symmetry plane condition is 


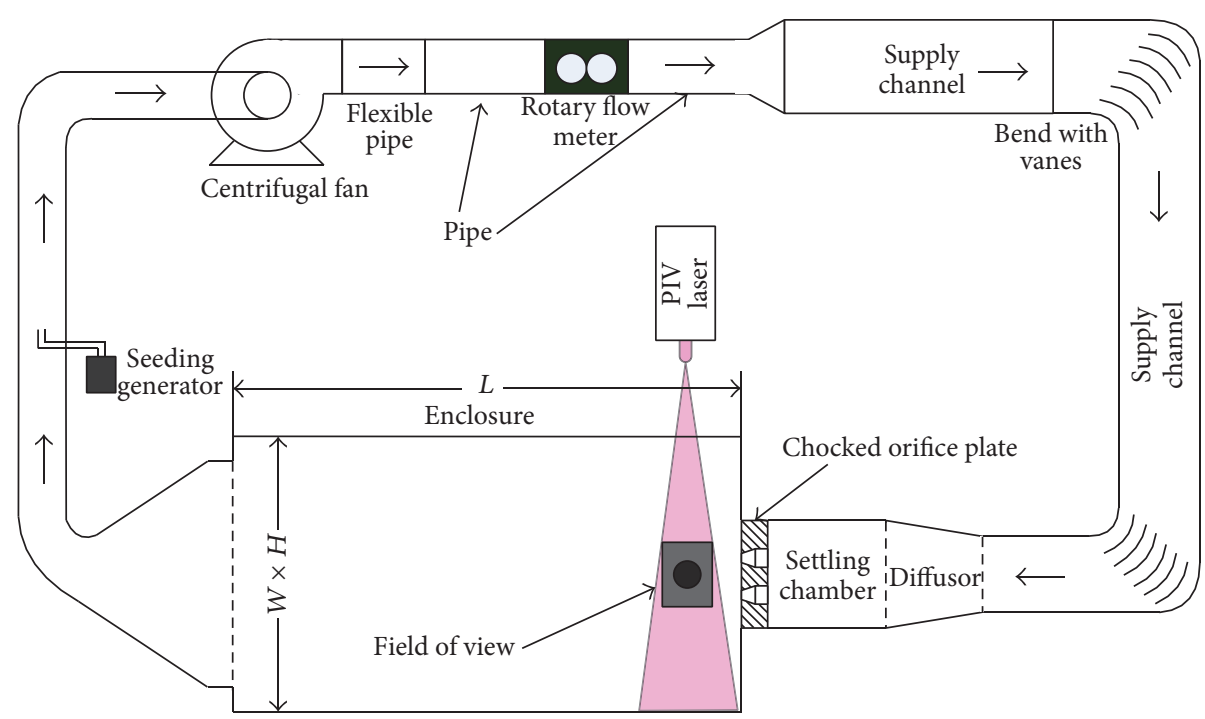

Figure 2: The schematic of experiment setup.

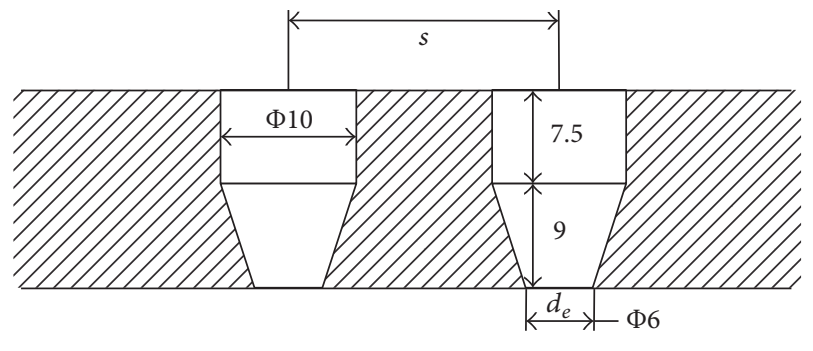

FIgURE 3: Detail of twin nozzles.

used on the walls parallel to the flow direction; and the pressure inlet boundary condition is applied to the nozzle inlet.

3.2. Governing Equation. Reynolds-Averaged Navier-Stokes Equations for three-dimensional compressible flows are given by

$$
\begin{aligned}
& \frac{\partial \rho}{\partial t}+\frac{\partial}{\partial x_{i}}\left(\rho u_{i}\right)=0 \\
& \frac{\partial}{\partial t}\left(\rho u_{i}\right)+\frac{\partial}{\partial x_{j}}\left(\rho u_{i} u_{j}\right) \\
& \quad=-\frac{\partial P}{\partial x_{i}}+\frac{\partial}{\partial x_{j}}\left(\mu \frac{\partial u_{i}}{\partial x_{j}}-\rho \overline{u_{i}^{\prime} u_{j}^{\prime}}\right), \\
& \frac{\partial}{\partial t}(\rho \phi)+\frac{\partial}{\partial x_{i}}\left(\rho u_{j} \phi\right)=\frac{\partial}{\partial x_{i}}\left(\Gamma \frac{\partial \phi}{\partial x_{i}}-\rho \overline{u_{i}^{\prime} \phi}\right)+S
\end{aligned}
$$

where the overbar indicates a time-averaged variable.
3.3. The RNG $k-\varepsilon$ Model. In this study, we employ the RNG $k-\varepsilon$ model for high Reynolds number flow given by Yakhot et al. [23]:

$$
\begin{aligned}
\frac{\partial}{\partial t}(\rho k)+\frac{\partial}{\partial x_{i}}\left(\rho k u_{i}\right)= & \frac{\partial}{\partial x_{j}}\left[\alpha_{k} \mu_{\mathrm{eff}} \frac{\partial k}{\partial x_{j}}\right]+\tau_{i j} \cdot S_{i j} \\
& -\rho \varepsilon \\
\frac{\partial}{\partial t}(\rho \varepsilon)+\frac{\partial}{\partial x_{i}}\left(\rho \varepsilon u_{i}\right)= & \frac{\partial}{\partial x_{j}}\left[\alpha_{\varepsilon} \mu_{\mathrm{eff}} \frac{\partial \varepsilon}{\partial x_{j}}\right]+C_{1 \varepsilon}^{*} \frac{\varepsilon}{k} \tau_{i j} \\
& \cdot S_{i j}-C_{2 \varepsilon} \rho \frac{\varepsilon^{2}}{k}
\end{aligned}
$$

with $\tau_{i j}=-\rho \overline{u_{i}^{\prime} u_{j}^{\prime}}=2 \mu_{t} S_{i j}-(2 / 3) \rho k \delta_{i j}, \mu_{\text {eff }}=\mu+\mu_{t}, \mu_{t}=$ $\rho C_{\mu}\left(k^{2} / \varepsilon\right), C_{\mu}=0.0845, \alpha_{k}=\alpha_{\varepsilon}=1.39, C_{1 \varepsilon}=1.42, C_{2 \varepsilon}=$ $1.68, C_{1 \varepsilon}^{*}=C_{1 \varepsilon}-\eta\left(1-\eta / \eta_{0}\right) /\left(1+\beta \eta^{3}\right), \eta=(k / \varepsilon) \sqrt{2 S_{i j} \cdot S_{i j}}$, $\beta=0.012, \eta_{0}=4.377$.

3.4. Numerical Scheme. The above transport equations have been solved using the standard FLUENT software in version 13.0 by Finite Volume Method. The implicit formulation and Roe-FDS flux type are for density-based solver type. The Green-Gauss Cell Based scheme is used for gradient discretization. Second-order upwind central differencing is used for flow terms, while first-order upwind central differencing is used for turbulent kinetic energy and turbulent dissipation rate. The solutions are considered to be converged when absolute values of the residuals are below $1 \times 10^{-5}$. The standard wall functions are used in the near-wall modeling.

3.5. Wall Yplus and Mesh Independency Tests. Figure 5 illustrates the computational mesh which consists of mesh, consists of structured hexahedral cells, and is refined closely to the wall, due to the high velocity gradients in this region. In order to eliminate grid quantity and quality effects on 


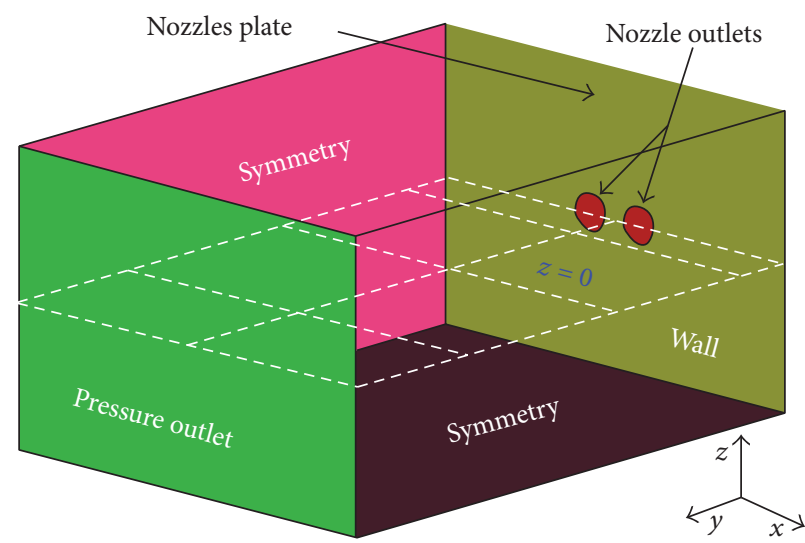

(a) Three-dimensional physical model
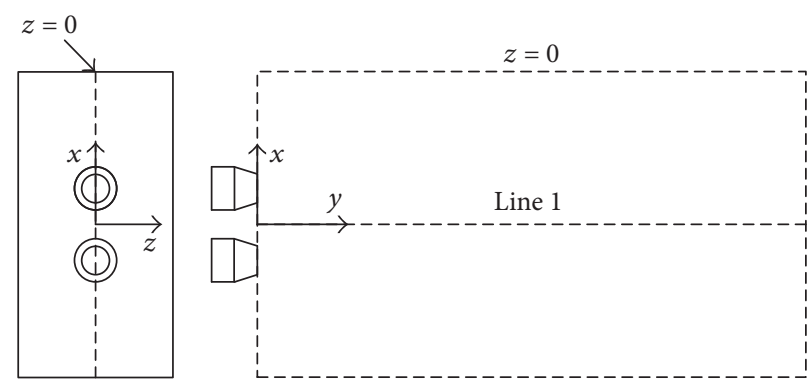

(b) Profiles of computational domain at $z=0$

FIgURE 4: The schematic diagram of computational domain.

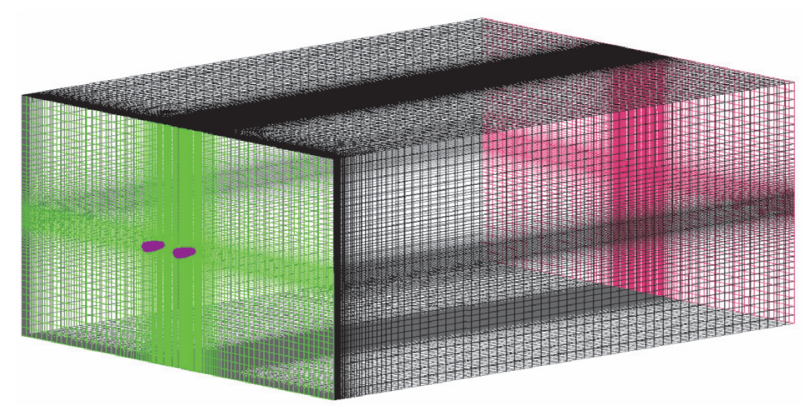

(a) The computational mesh

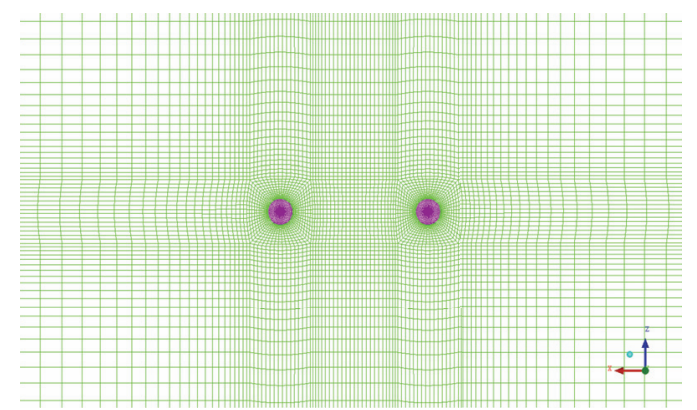

(b) Zoom-in of nozzles

FIGURE 5: The computational mesh and zoom-in of nozzles.

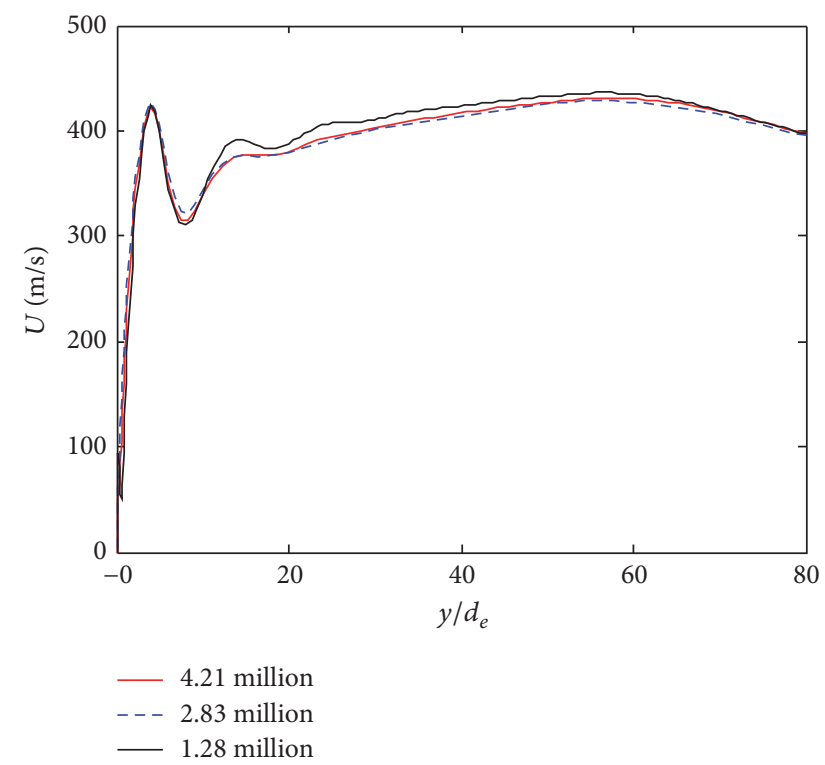

Figure 6: Axial mean velocity along downstream location.

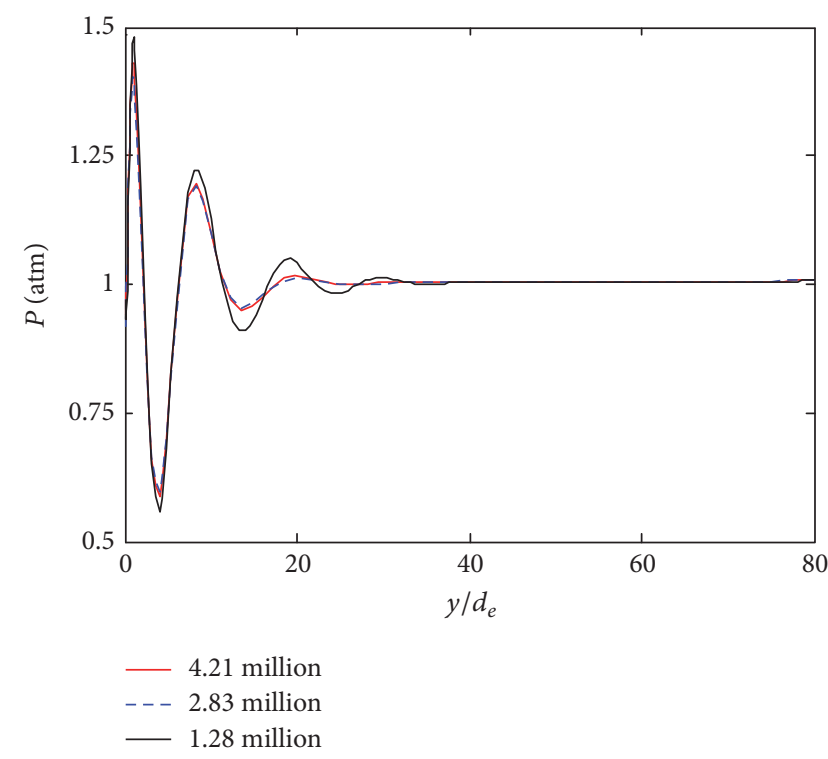

Figure 7: Axial mean pressure along downstream location. the numerical results, wall Yplus and mesh independency tests must be done using different mesh densities and grid configurations. Here, the mesh model $\left(s / d_{e}=2, P_{0} / P_{b}=\right.$ 6.8) was taken as an example to illustrate the wall Yplus and 


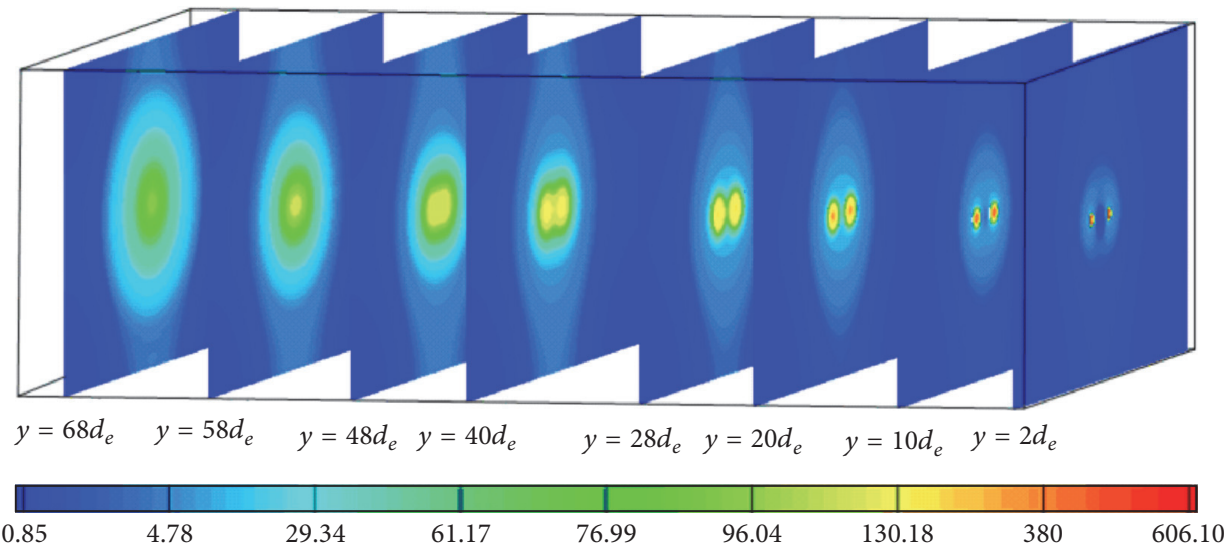

(a)
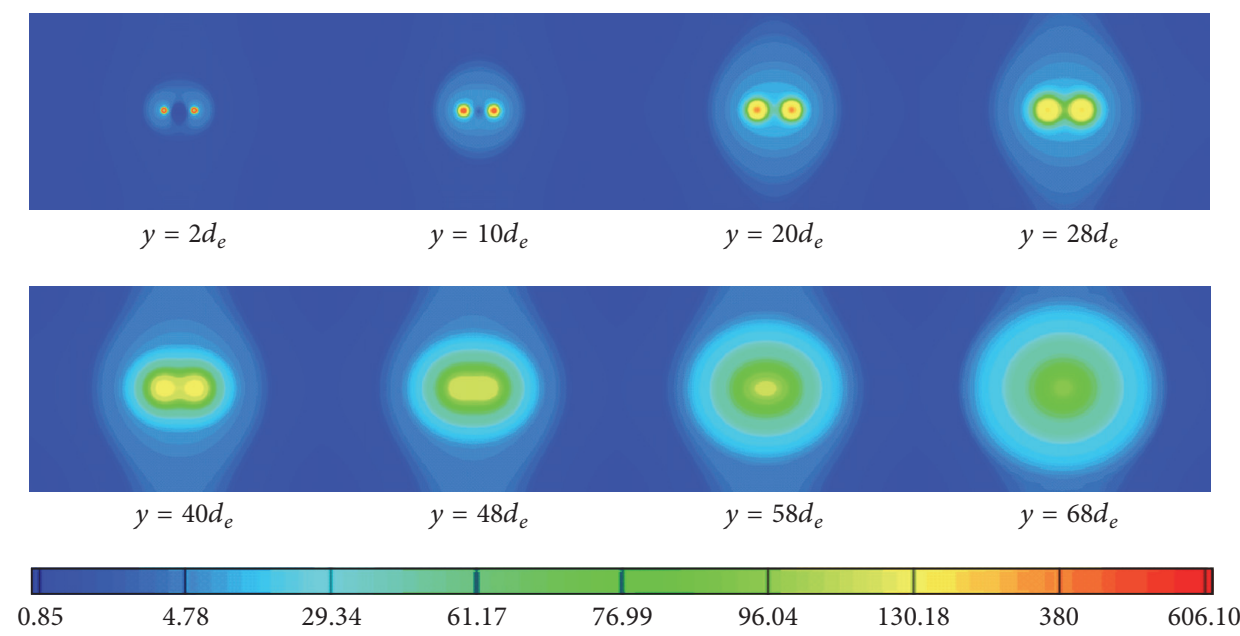

(b)

FIGURE 8: Contour of mean velocity along downstream cross-sectional planes numerical results.

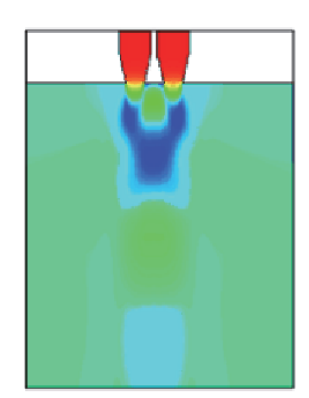

$s / d_{e}=2$

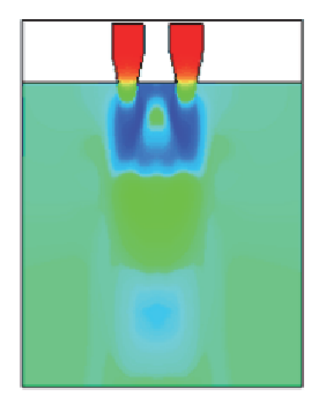

$s / d_{e}=3$

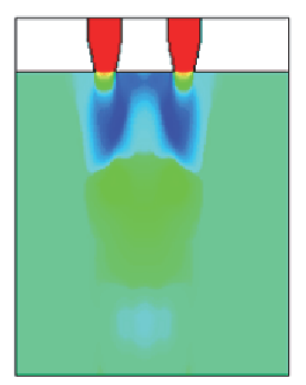

$s / d_{e}=4$

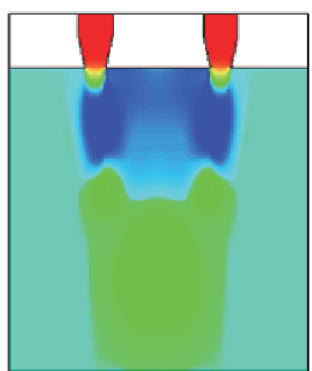

$s / d_{e}=6$

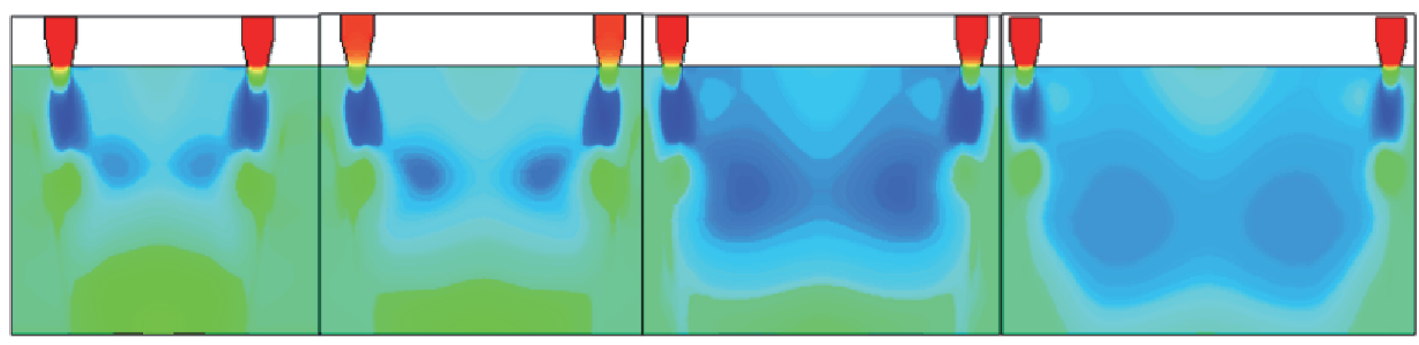

$s / d_{e}=20$

$s / d_{e}=10$

$s / d_{e}=13$

$s / d_{e}=16$

FIGURE 9: Contour of mean pressure along downstream cross-sectional planes numerical results at $P_{0} / P_{b}=6.8$. 

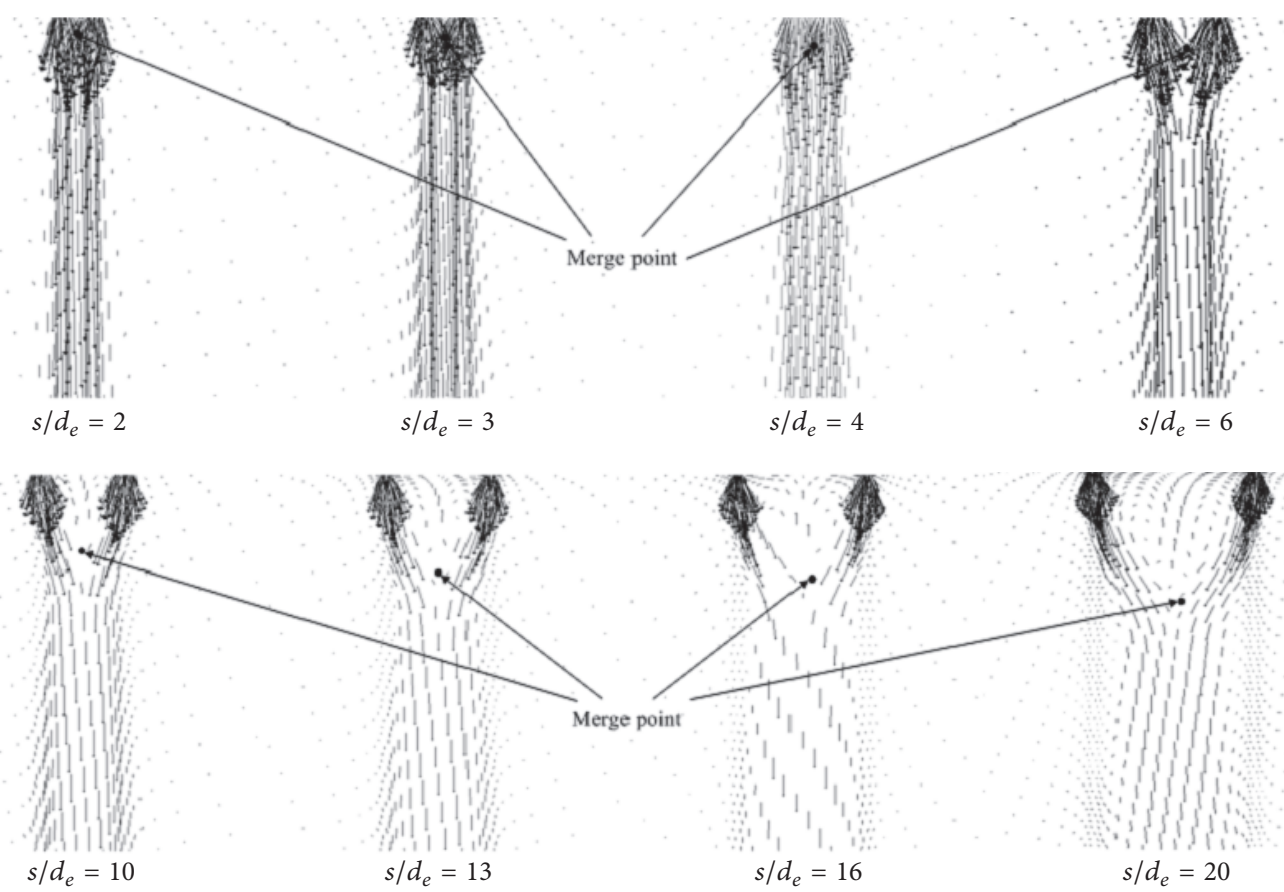

FIGURE 10: Vectors of mean velocity along downstream cross-sectional planes experimental results at $P_{0} / P_{b}=6.8$.
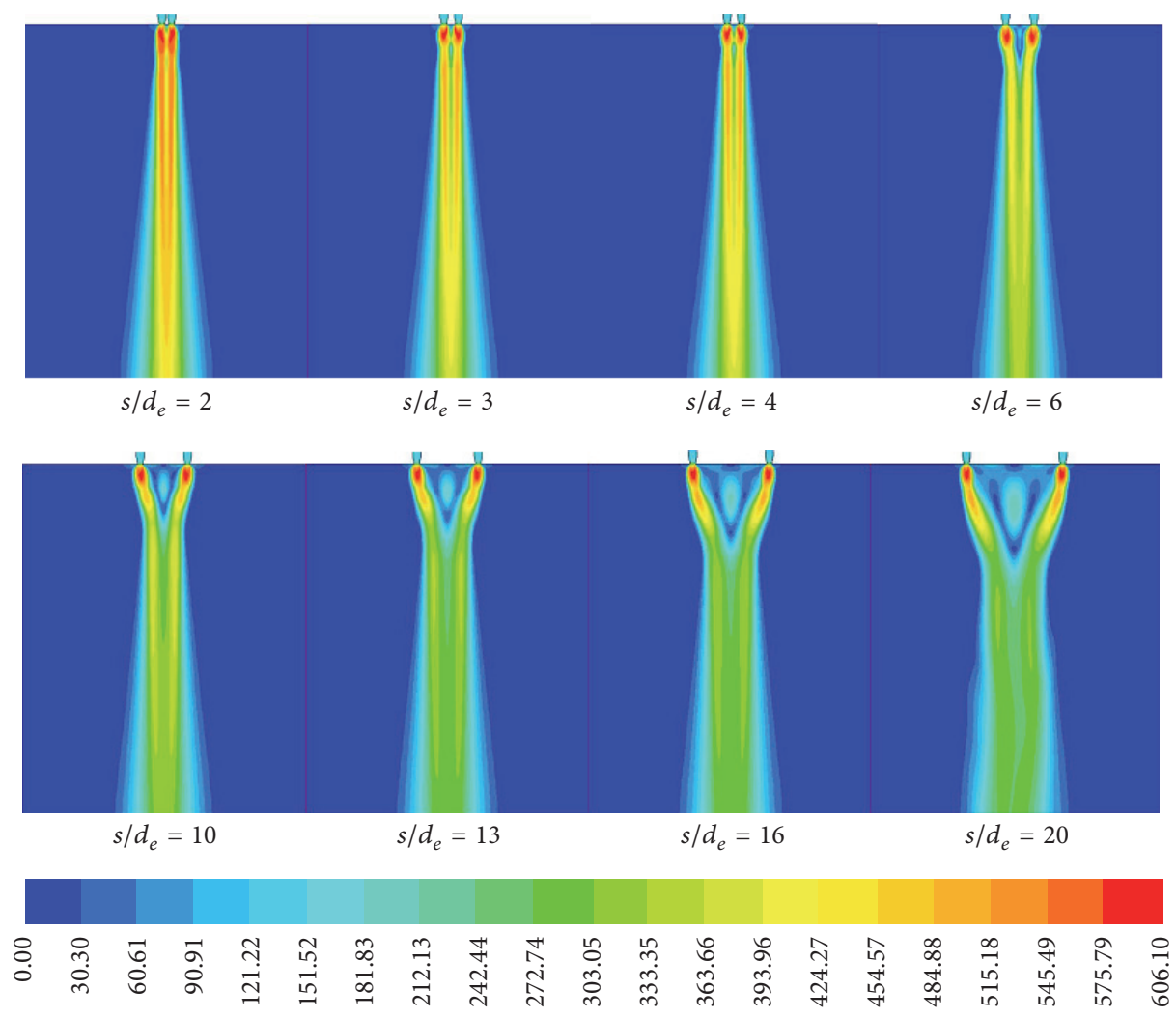

FIGURE 11: Contour of mean velocity along downstream cross-sectional planes numerical results at $P_{0} / P_{b}=6.8$. 

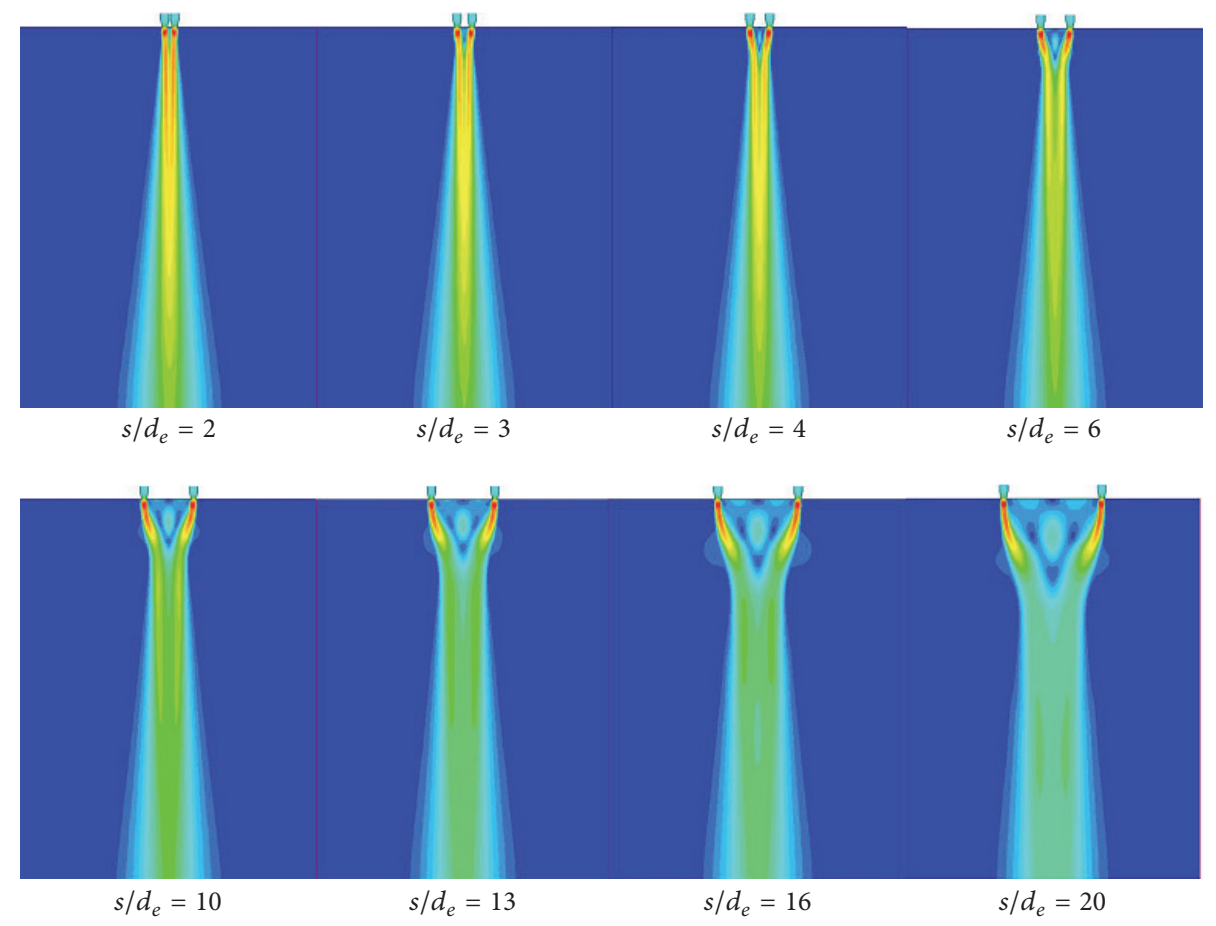

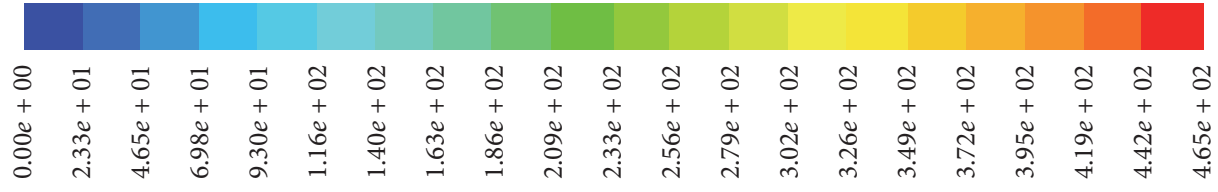

FIGURE 12: Contour of mean velocity along downstream cross-sectional planes numerical results at $P_{0} / P_{b}=3.4$.

mesh independency tests. For the standard wall functions, the wall Yplus values should range from 30 to 200. Based on the numerical results, the near-wall mesh density should be adjusted until the wall Yplus values are between 30 and 200. Figures 6 and 7 show the predictions of velocity and pressure at the line $x=0$ (on the plane $z=0$ ) along the flow field downstream, respectively. And the results are obtained on the three different mesh sizes, namely, 1.28 million, 2.83 million, and 4.11 million. The results difference between predictions of 1.28 million meshes and 2.83 million meshes is higher than the difference between 2.83 million meshes and 4.11 million meshes. It is indicated that the mesh size will slightly affect the numerical result when it is larger than 2.83 million. Therefore, the model with 2.83 million meshes' size is chosen to improve computing efficiency.

\section{Results and Discussions}

4.1. Characteristics of Double Jets. The characteristics profiles of mean velocity along the downstream location are presented in Figure 8. From Figure 8(a), we find that the double jets on both sides lean and bend towards the central symmetrical plane. Along the downstream location, the double jets gradually resemble a self-similar signal jet. Figure 8 demonstrates the velocity from numerical results at different downstream cross sections up to $y / d_{e}=80$. Figure 8 shows several features of the development of confluent jets. The faster merging of jets can be clearly observed; for example, $y / d_{e}=28$. At the nozzle exit, high velocities lead to entrainment of gas in the shear layers of the jets. Entrainment rates in the region between the two jets are high, which results in a region of very low pressure between the jets. The jets deflected towards each other. The deformation is found in all numerical results; and each of the models with different nozzle spacing and pressure ratio has its own specific features. Figure 8 shows the numerical results of the model with $s / d_{e}=6$ and $P_{0} / P_{b}=$ 6.8. Meanwhile, the experimental results also indicate the presence of jet deformation.

4.2. Effects of Jet Spacing. Tests 1 8 in Table 1 show the effect of various jet spacing on jet interaction. Figure 9 shows the contour of mean pressure along downstream cross-sectional planes numerical results at $P_{0} / P_{b}=6.8$. The numerical results show a subatmospheric pressure in the region between double nozzles, compared with that of their outside facing region. The negative pressure is related to the mutual entrainment between jets [24], and the subatmospheric pressure grows as strong as the jet spacing decreases. Therefore, the plume of each jet is shown to "bend" towards each other, because of the different pressure between mixed region and outward facing region of two jets. Figure 10 demonstrates the vectors of mean velocity at the downstream locations for eight different jet 


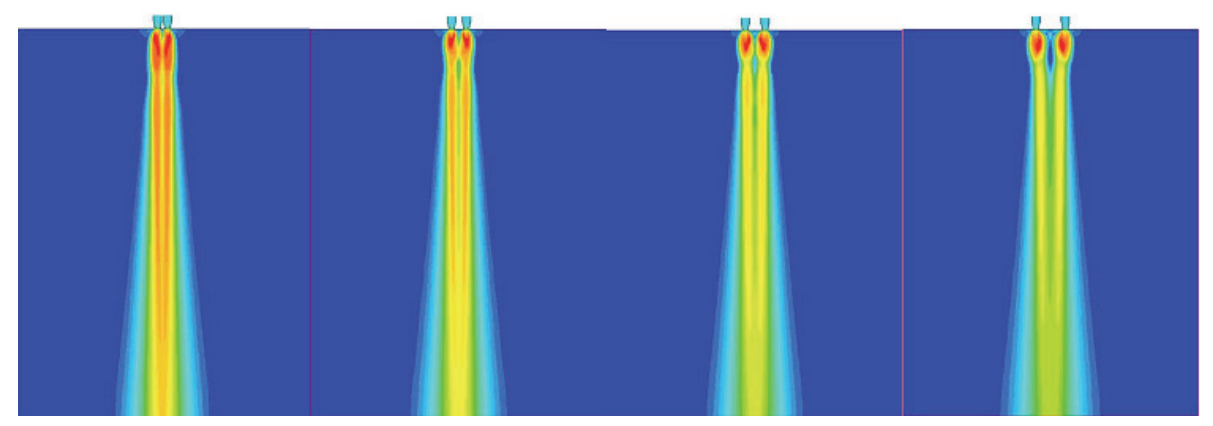

$s / d_{e}=2$

$s / d_{e}=3$

$s / d_{e}=4$

$s / d_{e}=6$

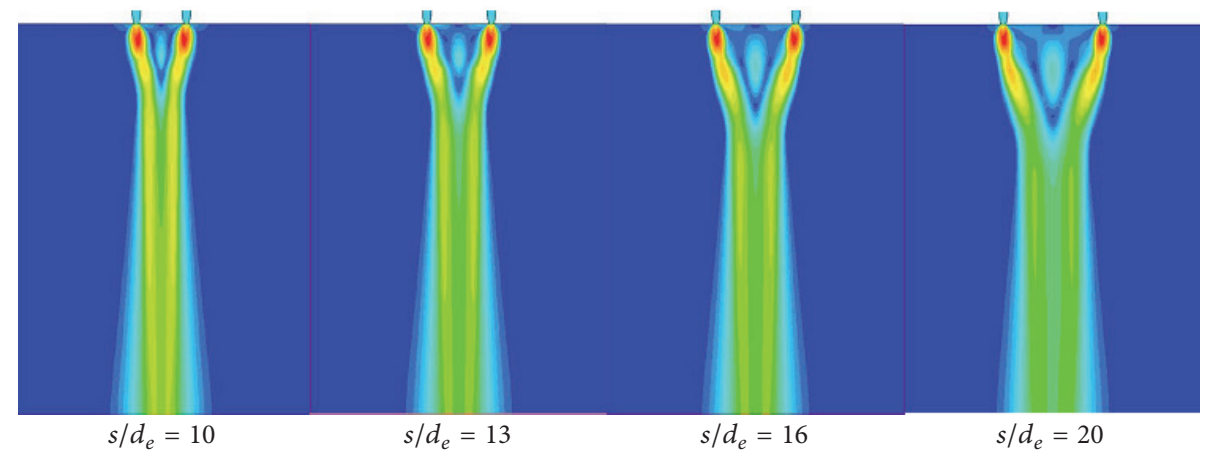

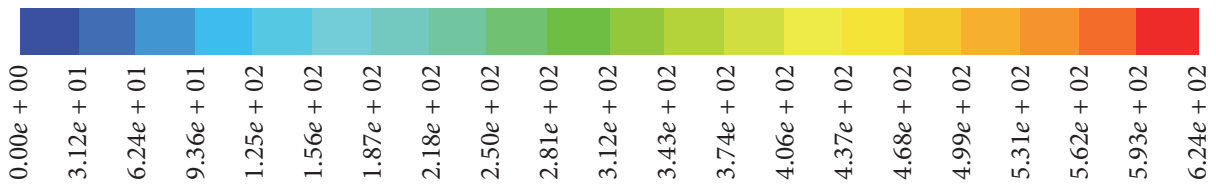

FIGURE 13: Contour of mean velocity along downstream cross-sectional planes numerical results at $P_{0} / P_{b}=8.1$.

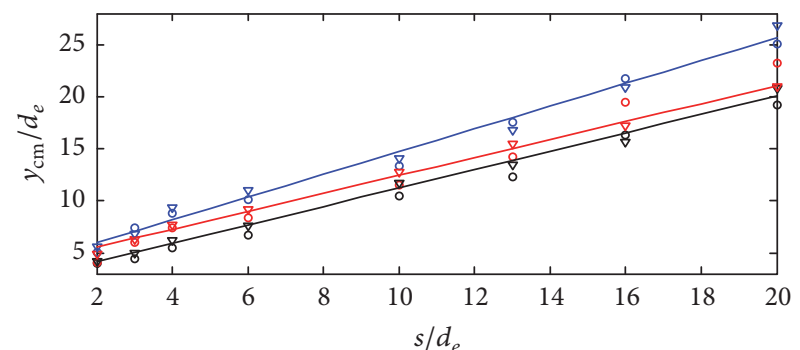

Experimental results Numerical results

$\begin{array}{ccc}\text { Equation (5) } & \nabla & \circ P_{0} / P_{b}=3.4 \\ - \text { Equation (3) } & \nabla & \circ P_{0} / P_{b}=6.8 \\ \text { Equation (7) } & \nabla & \circ P_{0} / P_{b}=8.1\end{array}$

(a) Merge point

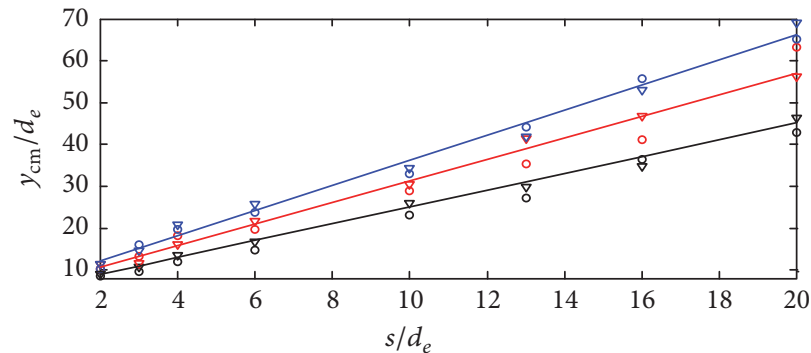

Experimental results Numerical results

$\begin{array}{lll}\text { Equation (6) } & \nabla & \circ P_{0} / P_{b}=3.4 \\ - \text { Equation (4) } & \nabla & \circ P_{0} / P_{b}=6.8 \\ \text { Equation (8) } & \nabla & \circ P_{0} / P_{b}=8.1\end{array}$

(b) Combine point

FIGURE 14: Variation in merge point and combine point for different spacing.

spacings. It is observed that the increased jets spacing shifts the merge point further downstream. The region from nozzle outlets $\left(y / D_{n}=0\right)$ to the merge point is the merging region, which is characterized by reverse flow in this region. From merge point inner shear layers of the jets begin to merge and are characterized as velocity is zero. The merge point is also characterized by high pressure. The contour of mean velocity along downstream cross-sectional plane (on the plane $z=0$ ) numerical results is presented in Figure 11, and the numerical results are consistent with its experimental results. The merge point locations and jets spacing are given in Table 2. We perform a regression analysis of the values shown in Table 2 to 


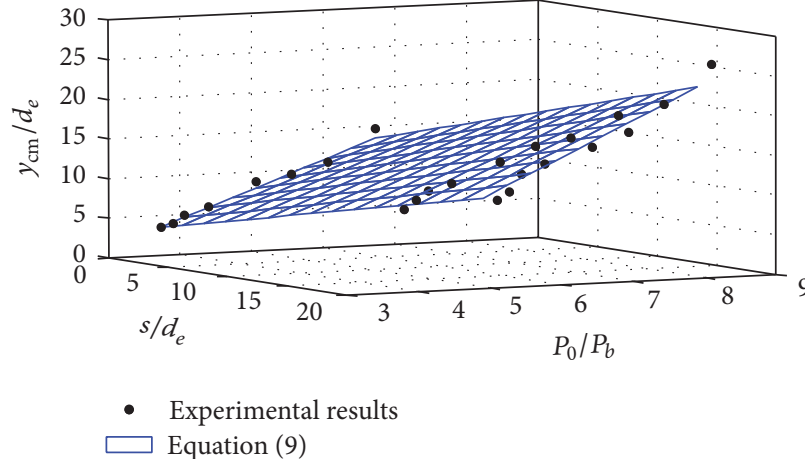

(a) Merge point

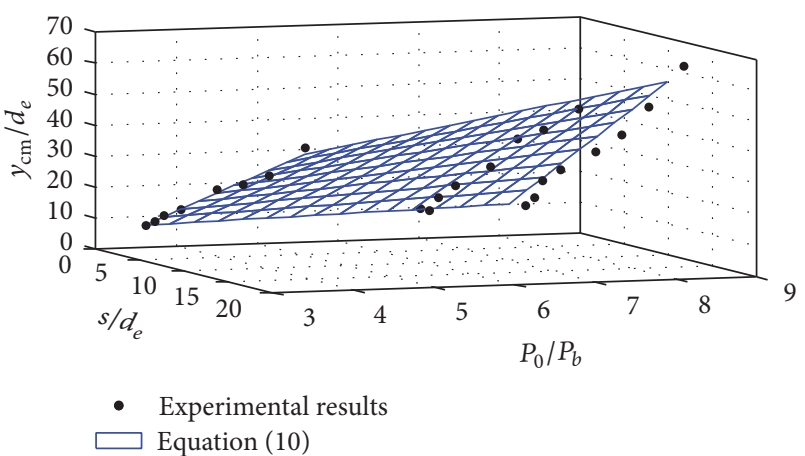

(b) Combine point

FIGURE 15: Variation in merge point and combine point for different spacing and pressure ratio.

discover the relationship between merge point locations and jets spacing. Regardless of the pressure ratio effect, we can get the following expressions:

$$
\begin{aligned}
& \frac{y_{\mathrm{MP}}}{d_{e}}=0.862\left(\frac{s}{d_{e}}\right)+3.824, \\
& \frac{y_{\mathrm{CP}}}{d_{e}}=2.578\left(\frac{s}{d_{e}}\right)+5.553 .
\end{aligned}
$$

The predictions based on (3) and (4) are presented in Figure 12. It can be observed that for numerical results and experimental results the relationship between the merge point and jet spacing is almost linear, so does the combine point. However, the slope of graph varies for merge point and combine point.

4.3. Effects of Pressure Ratio. In order to investigate the effect of pressure ratio, we carry out the tests with pressure ratios 3.4 and 8.1 shown in Table 1. It is a moderate underexpanded jet since $P_{0} / P_{b}$ is 3.4, which is less than 3.84, the criteria for a moderately underexpanded jet. Figures 12 and 13 are the contour of mean velocity along downstream cross-sectional planes (on the plane $z=0$ ) numerical results at different pressure ratio. As pressure ratio increases, the effect of jet interaction decreases, and the merge (combine) point location moves downstream. Detailed measurements for merge point and combine point locations are explained in Tables 3 and 4 . The merge point locations and jets spacing are given in Tables 3 and 4. Referring to Section 4.2, we can get the following expressions.

For pressure ratio 3.4, we have

$$
\begin{aligned}
& \frac{y_{\mathrm{MP}}}{d_{e}}=0.884\left(\frac{s}{d_{e}}\right)+2.396, \\
& \frac{y_{\mathrm{CP}}}{d_{e}}=2.011\left(\frac{s}{d_{e}}\right)+4.966 .
\end{aligned}
$$

For pressure ratio 8.1, we have

$$
\begin{aligned}
\frac{y_{\mathrm{MP}}}{d_{e}} & =1.094\left(\frac{s}{d_{e}}\right)+3.792, \\
\frac{y_{\mathrm{CP}}}{d_{e}} & =3.006\left(\frac{s}{d_{e}}\right)+6.131 .
\end{aligned}
$$

For the merge point, the relationship between the numerical results and the experimental results is given in Figures 14(a) and 15. It can be observed that for numerical results and experimental results the relationship between the merge point and jet spacing is almost linear. Meanwhile, the slope of graph varies with different pressure ratio, so does the combine point.

Figure 14(b) shows the location variation in combine point with different spacing.

We perform a regression analysis of the values shown in Tables 3 and 4 to discover the relationship between merge (combine) point locations, pressure ratio, and jets spacing. The following expressions can be obtained:

$$
\begin{aligned}
\frac{y_{\mathrm{MP}}}{d_{e}}= & 0.7371\left(\frac{s}{d_{e}}\right)+0.3279\left(\frac{P_{0}}{P_{b}}\right) \\
& +0.0343\left(\frac{P_{0}}{P_{b}}\right)\left(\frac{s}{d_{e}}\right)+1.3734, \\
\frac{y_{\mathrm{CP}}}{d_{e}}= & 1.2674\left(\frac{s}{d_{e}}\right)+0.2342\left(\frac{P_{0}}{P_{b}}\right) \\
& +0.2057\left(\frac{P_{0}}{P_{b}}\right)\left(\frac{s}{d_{e}}\right)+4.0803 .
\end{aligned}
$$

The prediction errors based on (9) and (10) are presented in Tables 3 and 4. It can be observed that the equations based on pressure ratio and jets spacing improve the prediction accuracy.

\section{Conclusions}

The mixing phenomenon of the dual underexpanded jets systems has been studied. The critical characteristics of 
TABLE 2: Predictions and errors of two points (merge point and combine point).

\begin{tabular}{|c|c|c|c|c|c|c|c|c|c|}
\hline \multicolumn{2}{|c|}{ Jet spacing (s) } & $2 d_{e}$ & $3 d_{e}$ & $4 d_{e}$ & $6 d_{e}$ & $10 d_{e}$ & $13 d_{e}$ & $16 d_{e}$ & $20 d_{e}$ \\
\hline \multirow{2}{*}{ Numerical results } & $\operatorname{MP}\left(y_{\mathrm{MP}} / d_{e}\right)$ & 4.13 & 6.02 & 7.39 & 8.37 & 11.51 & 14.27 & 19.52 & 23.22 \\
\hline & $\mathrm{CP}\left(y_{\mathrm{CP}} / d_{e}\right)$ & 10.15 & 13.15 & 18.27 & 19.59 & 28.81 & 35.40 & 41.20 & 63.17 \\
\hline \multirow{2}{*}{ Experimental results } & $\operatorname{MP}\left(y_{\mathrm{MP}} / d_{e}\right)$ & 4.92 & 6.30 & 7.71 & 9.13 & 12.73 & 15.47 & 17.20 & 20.92 \\
\hline & $\mathrm{CP}\left(y_{\mathrm{CP}} / d_{e}\right)$ & 11.38 & 11.50 & 16.15 & 21.52 & 30.43 & 41.42 & 46.65 & 56.16 \\
\hline \multicolumn{2}{|c|}{$y_{\mathrm{MP}} / d_{e}$ errors for numerical results (\%) } & 16.06 & 4.44 & 4.15 & 8.32 & 9.58 & 7.76 & 13.49 & 10.99 \\
\hline \multicolumn{2}{|c|}{$y_{\mathrm{CP}} / d_{e}$ errors for numerical results $(\%)$} & 10.81 & 14.35 & 13.13 & 8.97 & 6.34 & 14.53 & 11.68 & 12.48 \\
\hline \multicolumn{2}{|c|}{$y_{\mathrm{MP}} / d_{e}$ errors for $(3)(\%)$} & 12.76 & 1.75 & 5.68 & 1.47 & 2.25 & 2.84 & 2.42 & 0.69 \\
\hline \multicolumn{2}{|c|}{$y_{\mathrm{CP}} / d_{e}$ errors for $(4)(\%)$} & 5.90 & 15.54 & 1.76 & 2.32 & 2.97 & 5.68 & 0.32 & 1.70 \\
\hline \multicolumn{2}{|c|}{$y_{\mathrm{MP}} / d_{e}$ errors for $(9)(\%)$} & 12.68 & 3.40 & 2.93 & 3.23 & 4.53 & 4.83 & 11.21 & 9.99 \\
\hline \multicolumn{2}{|c|}{$y_{\mathrm{CP}} / d_{e}$ errors for $(10)(\%)$} & 3.29 & 18.88 & 1.16 & 0.70 & 6.26 & 2.62 & 3.60 & 5.05 \\
\hline
\end{tabular}

TABle 3: Predictions and errors of two points (merge point and combine point) at $P_{0} / P_{b}=3.4$.

\begin{tabular}{|c|c|c|c|c|c|c|c|c|c|}
\hline \multicolumn{2}{|c|}{ Jet spacing (s) } & $2 d_{e}$ & $3 d_{e}$ & $4 d_{e}$ & $6 d_{e}$ & $10 d_{e}$ & $13 d_{e}$ & $16 d_{e}$ & $20 d_{e}$ \\
\hline \multirow{2}{*}{ Numerical results } & $\operatorname{MP}\left(y_{\mathrm{MP}} / d_{e}\right)$ & 4.01 & 4.42 & 5.47 & 6.72 & 10.50 & 12.27 & 16.32 & 19.2 \\
\hline & $\mathrm{CP}\left(y_{\mathrm{CP}} / d_{e}\right)$ & 8.58 & 9.53 & 11.88 & 14.70 & 23.21 & 27.20 & 36.38 & 42.83 \\
\hline \multirow{2}{*}{ Experimental results } & $\operatorname{MP}\left(y_{\mathrm{MP}} / d_{e}\right)$ & 4.18 & 4.93 & 6.21 & 7.63 & 11.73 & 13.43 & 15.66 & 20.81 \\
\hline & $\mathrm{CP}\left(y_{\mathrm{CP}} / d_{e}\right)$ & 8.93 & 10.63 & 13.49 & 16.70 & 25.94 & 29.76 & 34.77 & 46.40 \\
\hline \multicolumn{2}{|c|}{$y_{\mathrm{MP}} / d_{e}$ errors for numerical results (\%) } & 4.07 & 10.34 & 11.92 & 11.93 & 10.49 & 8.64 & 4.21 & 7.74 \\
\hline \multicolumn{2}{|c|}{$y_{\mathrm{CP}} / d_{e}$ errors for numerical results (\%) } & 3.92 & 10.35 & 11.93 & 11.98 & 10.52 & 8.60 & 4.63 & 7.69 \\
\hline \multicolumn{2}{|c|}{$y_{\mathrm{MP}} / d_{e}$ errors for $(5)(\%)$} & 0.38 & 2.39 & 4.48 & 0.92 & 4.21 & 3.41 & 5.62 & 3.53 \\
\hline \multicolumn{2}{|c|}{$y_{\mathrm{CP}} / d_{e}$ errors for $(6)(\%)$} & 0.65 & 3.47 & 3.56 & 1.99 & 3.33 & 4.53 & 6.82 & 2.62 \\
\hline \multicolumn{2}{|c|}{$y_{\mathrm{MP}} / d_{e}$ errors for $(9)(\%)$} & 0.38 & 2.42 & 4.94 & 0.25 & 6.01 & 1.17 & 3.11 & 5.99 \\
\hline \multicolumn{2}{|c|}{$y_{\mathrm{CP}} / d_{e}$ errors for $(10)(\%)$} & 1.34 & 1.38 & 5.53 & 0.14 & 5.38 & 2.30 & 4.53 & 4.72 \\
\hline
\end{tabular}

mixing regions are predicted by jet spacing and pressure ratio. The models with different jets spacing and pressure ratio are simulated by FLUENT software and the numerical results coincide well with experimental results. Meanwhile, the following findings are obtained.

The two jets deflect towards inward facing region, and the deflection degree is determined by the jets spacing, pressure ratio, and turbulent intensity. Three typical regions (converging region, merging region, and combined region) are commonly reported. Along the downstream location, the two jets gradually resemble a self-similar signal jet.

The relationship between the merge point and jet spacing is almost linear; with different pressure ratio relationship is expressed by (3), (5), and (7). And (3), (5), and (7) would go as a criterion for design of various parallel jet systems such as cooling or boosting system.

The merge point location of two jet flows is affected by the jet spacing but also by the jet exit pressure ratio; and its relationship can be expressed by (9). And it is proved that the equation based on (9) can better its prediction accuracy.

The combine point location can be predicted by (4), (6), and (8).

\section{Nomenclature}

\section{Abbreviations}

PIV: Particle image velocimetry.
Symbols

$d_{e}: \quad \quad$ Nozzle exit diameter $(\mathrm{m})$

$H$ : $\quad$ Height of computational domain (m)

$L: \quad$ Length of computational domain (m)

$s: \quad \quad$ Nozzle spacing $(\mathrm{m})$

$P_{0}: \quad$ Total pressure (atm)

$P_{b}: \quad$ Back pressure (atm)

$W: \quad$ Width of computational domain (m)

$x_{i}(x, y, z)$ : Cartesian coordinates $(\mathrm{m})$

$C_{\mu}: \quad$ Turbulent viscosity constant

$y: \quad$ Streamwise coordinate $(\mathrm{m})$

$u_{1}, u_{1}^{\prime}$ : Mean and fluctuating velocity components, horizontal directions $\left(\mathrm{m} \cdot \mathrm{s}^{-1}\right)$

$u_{2}, u_{2}^{\prime}$ : Mean and fluctuating velocity component, streamwise direction $\left(\mathrm{m} \cdot \mathrm{s}^{-1}\right)$

$u_{3}, u_{3}^{\prime}$ : Mean and fluctuating velocity components, vertical directions $\left(\mathrm{m} \cdot \mathrm{s}^{-1}\right)$

$y_{\mathrm{MP}}: \quad$ Merge point location (m)

$y_{\mathrm{CP}}: \quad$ Combine point location $(\mathrm{m})$

$k: \quad$ Turbulent kinetic energy $\left(\mathrm{m}^{2} \cdot \mathrm{s}^{-2}\right)$

$\varepsilon$ : $\quad$ Rate of dissipation of turbulent kinetic energy $\left(\mathrm{m}^{2} \cdot \mathrm{s}^{-3}\right)$

$S: \quad$ Source terms

$C_{1 \varepsilon}, C_{2 \varepsilon}:$ Turbulence model coefficients

$\alpha_{k}, \alpha_{\varepsilon}: \quad$ Diffusion coefficients in turbulence models

$\delta_{i j}: \quad$ Kronecker delta

$\mu$ : $\quad$ Kinematic viscosity $\left(\mathrm{m}^{2} \cdot \mathrm{s}^{-1}\right)$ 
TABLE 4: Predictions and errors of two points (merge point and combine point) at $P_{0} / P_{b}=8.1$.

\begin{tabular}{|c|c|c|c|c|c|c|c|c|c|}
\hline \multicolumn{2}{|c|}{ Jet spacing (s) } & $2 d_{e}$ & $3 d_{e}$ & $4 d_{e}$ & $6 d_{e}$ & $10 d_{e}$ & $13 d_{e}$ & $16 d_{e}$ & $20 d_{e}$ \\
\hline \multirow{2}{*}{ Numerical results } & $\operatorname{MP}\left(y_{\mathrm{MP}} / d_{e}\right)$ & 5.06 & 7.41 & 8.79 & 10.11 & 13.35 & 17.55 & 21.72 & 25.11 \\
\hline & $\mathrm{CP}\left(y_{\mathrm{CP}} / d_{e}\right)$ & 10.29 & 15.97 & 19.69 & 23.72 & 32.90 & 44.25 & 55.63 & 65.14 \\
\hline \multirow{2}{*}{ Experimental results } & $\operatorname{MP}\left(y_{\mathrm{MP}} / d_{e}\right)$ & 5.61 & 6.83 & 9.36 & 11.01 & 14.05 & 16.73 & 20.89 & 26.81 \\
\hline & $\mathrm{CP}\left(y_{\mathrm{CP}} / d_{e}\right)$ & 11.33 & 14.55 & 20.80 & 25.63 & 34.33 & 41.74 & 52.95 & 68.98 \\
\hline \multicolumn{2}{|c|}{$y_{\mathrm{MP}} / d_{e}$ errors for numerical results (\%) } & 9.80 & 8.49 & 6.09 & 8.17 & 4.98 & 4.90 & 3.97 & 6.34 \\
\hline \multicolumn{2}{|c|}{$y_{\mathrm{CP}} / d_{e}$ errors for numerical results $(\%)$} & 9.18 & 9.76 & 5.34 & 7.45 & 4.17 & 6.01 & 5.06 & 5.57 \\
\hline \multicolumn{2}{|c|}{$y_{\mathrm{MP}} / d_{e}$ errors for $(7)(\%)$} & 6.60 & 3.57 & 12.74 & 5.94 & 4.85 & 7.67 & 1.94 & 4.24 \\
\hline \multicolumn{2}{|c|}{$y_{\mathrm{CP}} / d_{e}$ errors for $(8)(\%)$} & 7.18 & 4.12 & 12.72 & 5.71 & 5.42 & 8.31 & 2.41 & 3.96 \\
\hline \multicolumn{2}{|c|}{$y_{\mathrm{MP}} / d_{e}$ errors for (9) (\%) } & 8.01 & 3.58 & 13.58 & 8.09 & 0.92 & 2.95 & 2.98 & 9.26 \\
\hline \multicolumn{2}{|c|}{$y_{\mathrm{CP}} / d_{e}$ errors for $(10)(\%)$} & 4.54 & 1.57 & 14.85 & 8.0 & 2.86 & 5.69 & 0.67 & 6.28 \\
\hline
\end{tabular}

$\mu_{t}$ : Turbulent viscosity $\left(\mathrm{m}^{2} \cdot \mathrm{s}^{-1}\right)$

$\rho$ : Jet density $\left(\mathrm{kg} \cdot \mathrm{m}^{-3}\right)$

$S_{i j}:$ Rate of strain tensor $\left(\mathrm{s}^{-1}\right)$

$\tau_{i j}:$ Stress vector $\left(\mathrm{kg} \cdot \mathrm{m}^{-1} \cdot \mathrm{s}^{-2}\right)$

$\eta$ : Ratio of the turbulent to mean strain time scale.

\section{Competing Interests}

The authors declare that they have no competing interests.

\section{Acknowledgments}

The authors gratefully acknowledge the financial support received from National Natural Science Foundation of China (51475462).

\section{References}

[1] Y. Cho, H. B. Awbi, and T. Karimipanah, "Theoretical and experimental investigation of wall confluent jets ventilation and comparison with wall displacement ventilation," Building and Environment, vol. 43, no. 6, pp. 1091-1100, 2008.

[2] S. Janbakhsh, B. Moshfegh, and S. Ghahremanian, "A newly designed supply diffuser for industrial premises," International Journal of Ventilation, vol. 9, no. 1, pp. 59-67, 2010.

[3] K. Svensson, P. Rohdin, B. Moshfegh, and M. J. Tummers, "Numerical and experimental investigation of the near zone flow field in an array of confluent round jets," International Journal of Heat and Fluid Flow, vol. 46, pp. 127-146, 2014.

[4] E. A. Anderson and R. E. Spall, "Experimental and numerical investigation of two-dimensional parallel jets," Journal of Fluids Engineering, Transactions of the ASME, vol. 123, no. 2, pp. 401406, 2001.

[5] Z. Baratian-Ghorghi, N. B. Kaye, A. A. Khan, and J. R. Smith, "The merging of two unequal axisymmetric parallel turbulent jets," Journal of Hydrodynamics, vol. 24, no. 2, pp. 257-262, 2012.

[6] A. Durve, A. W. Patwardhan, I. Banarjee, G. Padmakumar, and G. Vaidyanathan, "Numerical investigation of mixing in parallel jets," Nuclear Engineering and Design, vol. 242, pp. 78-90, 2012.

[7] D. R. Miller and E. W. Comings, "Force-momentum fields in a dual-jet flow," Journal of Fluid Mechanics, vol. 7, no. 2, pp. 237256,1960 .
[8] E. Tanaka, "The interference of two-dimensional parallel jets," Bulletin JSME, vol. 13, no. 56, pp. 272-280, 1970.

[9] E. Tanaka, "The interference of two-dimensional parallel jets," Bulletin of the JSME, vol. 17, pp. 920-927, 1974.

[10] A. Vouros and T. Panidis, "Influence of a secondary, parallel, low Reynolds number, round jet on a turbulent axisymmetric jet," Experimental Thermal and Fluid Science, vol. 32, no. 8, pp. 1455-1467, 2008.

[11] H. Elbanna and J. A. Sabbagh, "Interaction of two nonequal plane parallel jets," AIAA Journal, vol. 25, no. 1, pp. 12-13, 1987.

[12] R. E. Spall, "Numerical study of buoyant plane parallel jets," Journal of Heat Transfer, vol. 124, no. 6, pp. 1210-1212, 2002.

[13] D. Tenchine and J. P. Moro, "Experimental and numerical study of coaxial jets," in Proceedings of the 8th International Topical Meeting on Nuclear Reactor Thermal-Hydraulics, vol. 3, pp. 13811387, Kyoto, Japan, 1997.

[14] Q. Cao, D. Lu, and J. Lv, "Numerical investigation on temperature fluctuation of the parallel triple-jet," Nuclear Engineering and Design, vol. 249, pp. 82-89, 2012.

[15] A. Tokuhiro and N. Kimura, "Experimental investigation on thermal striping. Mixing phenomena of a vertical non-buoyant jet with two adjacent buoyant jets as measured by ultrasound Doppler velocimetry," Nuclear Engineering and Design, vol. 188, no. 1, pp. 49-73, 1999.

[16] N. Kimura, M. Igarashi, and H. Kamide, "Investigation on convective mixing of triple jet evaluation of turbulent quantities using particle image velocimetry and direct numerical simulation," in Proceedings of the 8th International Symposium on Flow Modeling and Turbulence Measurements, pp. 651-658, Tokyo, Japan, 2001.

[17] T. Okamoto, M. Yagita, A. Watanabe, and K. Kawamura, "Interaction of twin turbulent circular jet," Bulletin of the JSME, vol. 28, no. 238, pp. 617-622, 1985.

[18] J. B. Allen and D. L. Smith, "Numerical modeling of turbulent, parallel, round jets," in Proceedings of the DoD High Performance Computing Modernization Program Users Group Conference (HPCMP UGC '10), pp. 3-7, Schaumburg, Ill, USA, June 2010.

[19] T. Harima, S. Fujita, and H. Osaka, "Turbulent properties of twin circular free jets with various nozzle spacing," Engineering Turbulence Modelling and Experiments, vol. 6, pp. 501-510, 2005.

[20] T.-S. Wang, "Numerical analysis of base flowfield for a fourengine clustered nozzle configuration," Journal of Propulsion and Power, vol. 11, no. 5, pp. 1076-1078, 1995. 
[21] M. Stanislas, K. Okamoto, C. J. Kähler, J. Westerweel, and F. Scarano, "Main results of the third international PIV Challenge," Experiments in Fluids, vol. 45, no. 1, pp. 27-71, 2008.

[22] R. J. Adrian and J. Westerweel, Particle Image Velocimetry, Cambridge University Press, Cambridge, UK, 2010.

[23] V. Yakhot, S. A. Orszag, S. Thangam, T. B. Gatski, and C. G. Speziale, "Development of turbulence models for shear flows by a double expansion technique," Physics of Fluids A. Fluid Dynamics, vol. 4, no. 7, pp. 1510-1520, 1992.

[24] I. Yimer, H. A. Becker, and E. W. Grandmaison, "Development of flow from multiple-jet burners," Canadian Journal of Chemical Engineering, vol. 74, no. 6, pp. 840-851, 1996. 


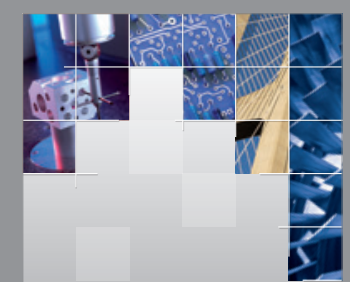

\section{Enfincering}
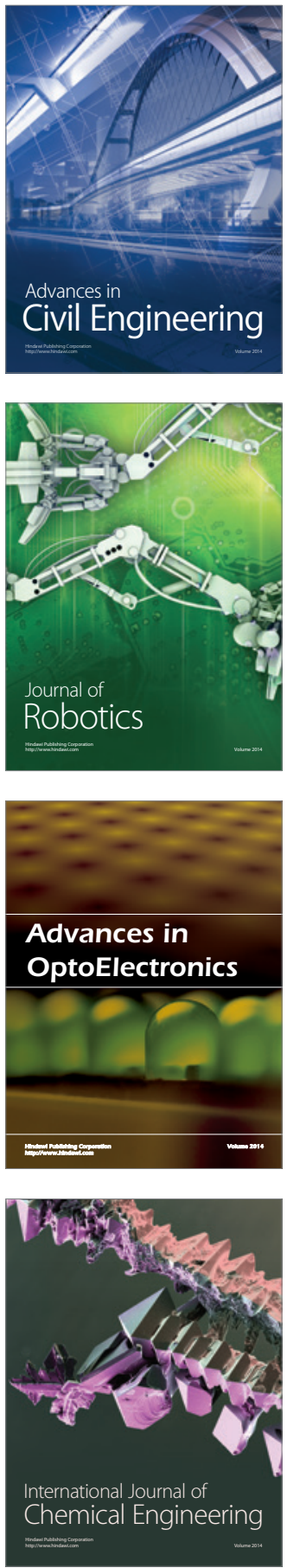

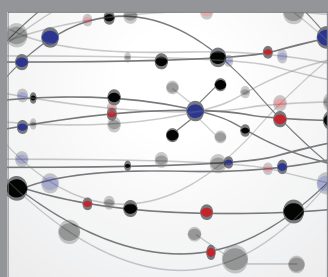

The Scientific World Journal

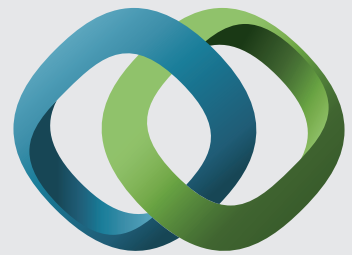

\section{Hindawi}

Submit your manuscripts at

http://www.hindawi.com
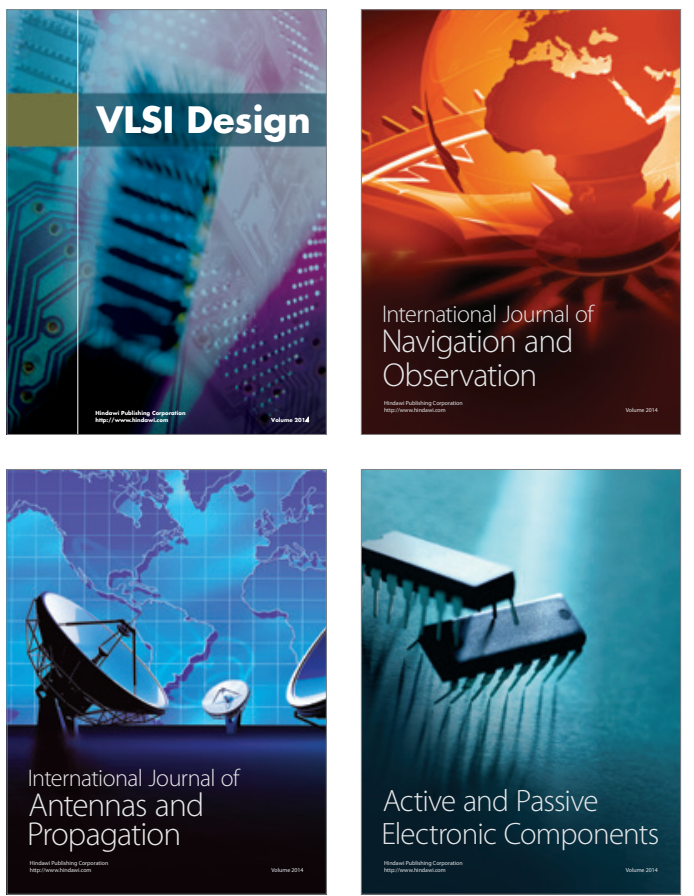
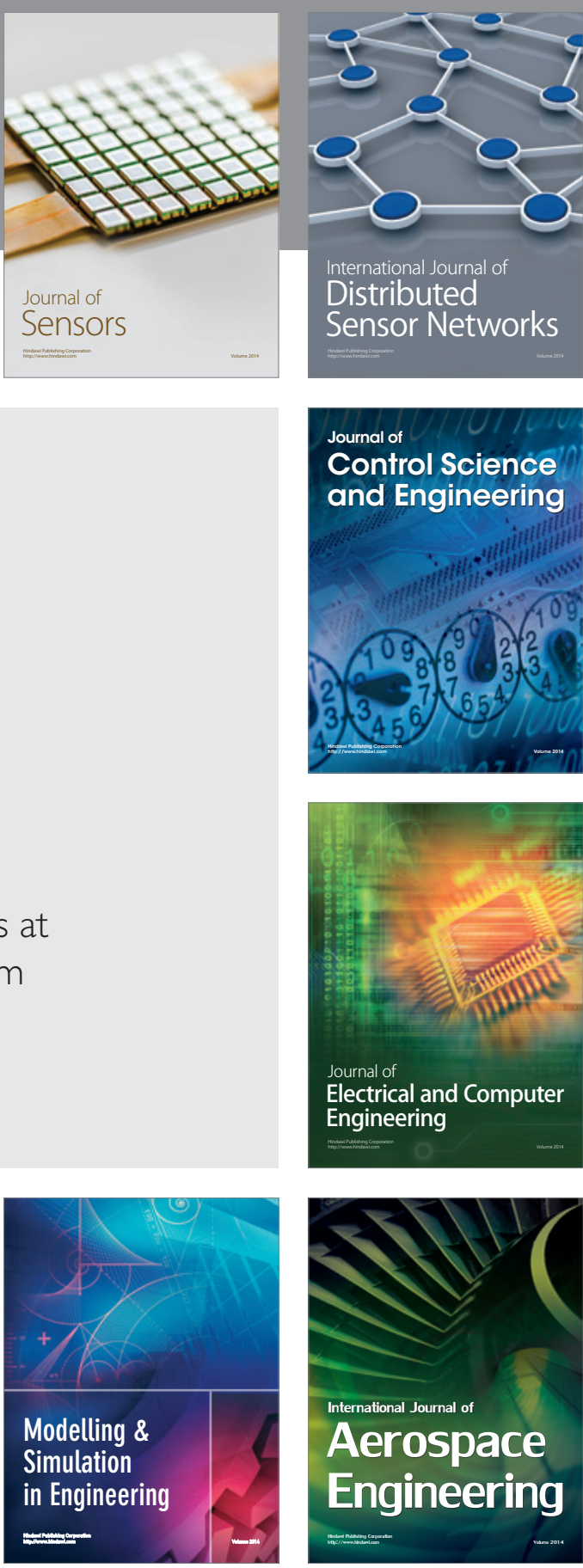

International Journal of

Distributed

Sensor Networks

Journal of

Control Science

and Engineering
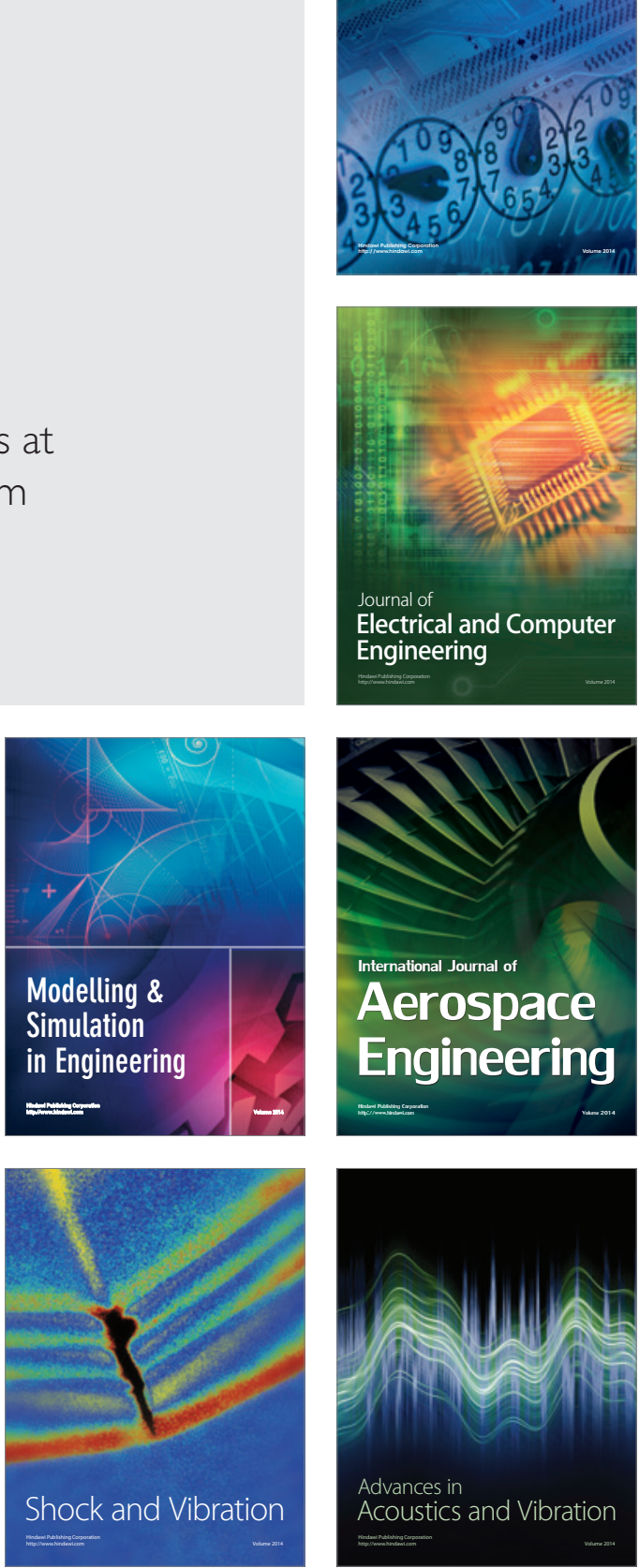